\title{
MAINTAINING MINIMUM SPANNING FORESTS IN DYNAMIC GRAPHS*
}

\author{
MONIKA R. HENZINGER ${ }^{\dagger}$ AND VALERIE KING‡
}

\begin{abstract}
We present the first fully dynamic algorithm for maintaining a minimum spanning forest in time $o(\sqrt{n})$ per operation. To be precise, the algorithm uses $O\left(n^{1 / 3} \log n\right)$ amortized time per update operation. The algorithm is fairly simple and deterministic. An immediate consequence is the first fully dynamic deterministic algorithm for maintaining connectivity and bipartiteness in amortized time $O\left(n^{1 / 3} \log n\right)$ per update, with $O(1)$ worst case time per query.
\end{abstract}

Key words. dynamic graph, graph algorithm, minimum spanning tree, data structure

AMS subject classifications. 05C85, 68W40, 68Q25, 68P05

PII. S0097539797327209

1. Introduction. We consider the problem of maintaining a minimum spanning forest during an arbitrary sequence of edge insertions and deletions. Given an $n$-vertex graph $G$ with edge weights, the fully dynamic minimum spanning forest problem is to maintain a minimum spanning forest $F$ under an arbitrary sequence of the following update operations:

insert $(u, v)$. Add the edge $\{u, v\}$ to $G$. Add $\{u, v\}$ to $F$ if it connects two previously unconnected trees of $F$ or if it reduces the cost of $F$. If the latter, return the edge of $F$ that has been replaced.

delete $(u, v)$. Remove the edge $\{u, v\}$ from $G$. If $\{u, v\} \in F$, then (a) remove $\{u, v\}$ from $F$ and (b) return the minimum-cost edge $e$ of $G \backslash F$ that reconnects $F$ if $e$ exists or return null if $e$ does not exist.

In addition, the data structure permits the following type of query:

connected $(u, v)$. Determine if vertices $u$ and $v$ are connected.

In 1985 [7], Fredrickson introduced a data structure known as topology trees for the fully dynamic minimum spanning forest problem with a worst-case cost of $O(\sqrt{m})$ per update His data structure permitted connectivity queries to be answered in $O(1)$ time. In 1992, Eppstein, Galil, and Italiano [3] and Eppstein et al. [4] improved the update time to $O(\sqrt{n})$ using the sparsification technique. If only edge insertions are allowed, the Sleator-Tarjan dynamic tree data structure [13] can be used to maintain the minimum spanning forest in time $O(\log n)$ per insertion or query. If only edge deletions are allowed ("deletions-only"), then no algorithm faster than the $\Omega(\sqrt{n})$ fully dynamic algorithm is known.

Using randomization, it was recently shown that the fully dynamic connectivity problem, i.e., the restricted problem where all edge costs are the same, can be solved in amortized time $O\left(\log ^{2} n\right)$ per update and $O(\log n)$ per connectivity query $[9,10]$. However, this approach could not be extended to arbitrary edge weights, leaving the question open as to whether the fully dynamic minimum spanning forest problem can be solved in time $o(\sqrt{n})$.

${ }^{*}$ Received by the editors September 16, 1997; accepted for publication (in revised form) December 19, 2000; published electronically August 8, 2001. A preliminary version of this work appeared in ICALP' 97.

http://www.siam.org/journals/sicomp/31-2/32720.html

${ }^{\dagger}$ Google, Inc., Mountain View, CA (monika@google.com).

${ }^{\ddagger}$ Department of Computer Science, University of Victoria, Victoria, BC, Canada V8W 3P4 (val@csr.uvic.ca). The research of this author was supported by an NSERC grant. 
In this paper we give a positive answer to this question: We present a fully dynamic minimum spanning forest data structure that uses $O\left(n^{1 / 3} \log n\right)$ amortized time per update and $O(1)$ worst case time per query when update time is averaged over any sequence of $\Omega\left(m_{i n}\right)$ updates for $m_{i n}$ the initial size of the graph. Our technique is very different from [7].

The result is achieved in two steps: First, we give a deletions-only minimum spanning forest algorithm that uses $O\left(m^{\prime 1 / 3} \log n+n^{\epsilon}\right)$ amortized time per update and $O(1)$ worst case time per query when the update time is averaged over any sequence of $\Omega\left(m_{i n}\right)$ updates. Here $\epsilon$ is any constant such that $0<\epsilon<1 / 3$, and $m^{\prime}$ is the number of nontree edges at the time of the update.

Then we present a general technique which, given a deletions-only minimum spanning forest data structure with a certain property, generates a fully dynamic data structure with the same running time as the deletions-only data structure. Let $f\left(m^{\prime}, n\right)$ be the amortized time per deletion in the deletions-only data structure with $m^{\prime}$ nontree edges and $n$ vertices. The property required is that, upon inserting into the graph no more than $m^{\prime}$ edges at the same time (a "batch insertion"), the deletionsonly data structure can be modified to reflect these insertions and up to $m^{\prime}$ subsequent deletions can be performed in a total of $O\left(m^{\prime} f\left(m^{\prime}, n\right)\right)$ time.

Using this technique, we develop a fully dynamic minimum spanning forest algorithm with amortized time per update of $O\left(m^{1 / 3} \log n\right)$ for a sequence of updates of length $\Omega\left(m_{i n}\right)$, where $m$ is the size of $G$ at the time of the update. In other words, letting $m_{(i)}$ denote the size of $G$ (vertices and edges) after update $i$, the total amount of work for processing a sequence of updates of length $l$ is $O\left(\sum_{i=0}^{l} m_{(i)}{ }^{1 / 3} \log n\right)$. We then apply sparsification $[3,4]$ to reduce the running time for the sequence to $O\left(l n^{1 / 3} \log n\right)$.

Our result immediately gives faster deterministic fully dynamic algorithms for the following problems: connectivity, bipartiteness, $k$-edge witness, maximal spanning forest decomposition, and Euclidean minimum spanning tree. See [9] for all but the last reduction; see Eppstein [2] for the last reduction. For these problems, the new algorithm achieves an $O\left(n^{1 / 6} / \log n\right)$ factor improvement over the previously best deterministic running time. If randomization is allowed, however, much faster times are achievable $[9,10]$.

Additionally, improvements can be achieved in the following static problems (see $[4,3])$ : randomly sampling spanning forests of a given graph [6] and finding a color-constrained minimum spanning tree [8].

The paper is structured as follows: In section 2 we give a deletions-only minimum spanning forest algorithm. In section 3, we show how to use a sequence of deletionsonly data structures to create a fully dynamic data structure.

2. Maintaining a minimum spanning forest-Deletions-only. In this section, we give an algorithm which maintains a minimum spanning forest while edges are being deleted. The amortized update time is $O\left(m^{1 / 3} \log n\right)$ and the query time is $O(1)$ for queries of the form "Are vertices $i$ and $j$ connected?". Let $G=(V, E)$ be an undirected graph with edge weights. Without loss of generality, we assume that edge weights are distinct.

Initially, we compute the minimum spanning forest $F$ of $G$. Let $m_{i n}^{\prime}$ be the number of nontree edges in $G$ initially and $k=m^{\prime 1 / 3} \log n$. We sort the nontree edges by weight and partition them into $m^{\prime}{ }_{i n} / k$ levels of size $k$ so that the $k$ lightest are in level 0 , the next $k$ lightest are in level 1 and so on. The set of edges in a level $i$ is denoted by $E_{i}$. In addition, all tree edges of the initial minimum spanning forest $F$ 
are placed in level 0 . (We omit floors and ceilings to simplify notation; either can be used without affecting the asympotic analysis.)

Throughout the algorithm, the level of an edge remains unchanged, and $F$ denotes the minimum spanning forest. For $i=0,1, \ldots,\left(m^{\prime}{ }_{i n} / k\right)-1$, let $F_{i}$ denote the minimum spanning forest of the graph with vertex set $V$ and edgeset $\cup_{j \leq i} E_{j}$. (Initially, all $F_{i}=F$, but in later stages, an edge from any level may become a tree edge. Thus, $F_{0} \subseteq F_{1} \subseteq \ldots \subseteq F_{\left(m^{\prime} 0 / k\right)-1}=F$.) Let $T_{i}(x)$ denote the tree in $F_{i}$ which contains $x$ and let $T(x)$ without the subscript denote the tree in $F$ containing $x$.

The main idea is the following. If a nontree edge is deleted, then the minimum spanning forest $F$ is unchanged. Suppose a tree edge $\{u, v\}$ in level $i$ is deleted. Then for each $F_{j}, j \geq i$, the deletion splits the tree in $F_{j}$ containing $u$ and $v$ into $T_{j}(u)$ and $T_{j}(v)$. We search for the minimum weight nontree edge $e$ (called the "replacement edge") that connects $T(u)$ and $T(v)$ by gathering and then testing a set $S$ of candidate edges on level $i$. If none is found, we repeat the procedure on level $i+1$, etc., until one is found or all levels are exhausted.

We now describe the update operations.

$\operatorname{delete}(u, v)$. Delete edge $\{u, v\}$ from any data structures in which it occurs. If a tree edge $\{u, v\}$ from level $i$ is deleted, then remove $\{u, v\}$ from $F$ and search for a replacement by calling $\operatorname{Replace}(i, u, v)$. We refer to $i$ as the level of the call to Replace.

In the algorithm below, the subroutine Search when applied to a tree in $F_{i}$ finds all nontree edges in level $i$ which are incident to the tree. A phase consists of the examination of a single edge. (Its exact definition and the details of Search are given in section 2.2 below.)

Replace $(i, u, v)$.

1. Alternating in lockstep, one phase at a time, $\operatorname{Search}\left(T_{i}(u)\right)$ and $\operatorname{Search}\left(T_{i}(v)\right)$ until $k / \log n$ phases are executed (Case A) or one of the searches has stopped (Case B).

- Case A: Let $S$ be the set of all nontree edges in level $i$.

- Case B: Let $S$ be the set of (nontree) edges produced by the Search that stopped.

2. Test every edge in $S$ to see if it connects $T(u)$ and $T(v)$.

- If a connecting edge is found, insert the minimum weight connecting edge into $F$ and the data structures representing the $F_{j}, j \geq i$.

- Else if $i$ is not the last level, call $\operatorname{Replace}(i+1, u, v)$.

2.1. Data structures. The idea here is to use the ET-tree data structure developed in [9]: (1) to represent and update each tree in $F$, so that in constant time, we can quickly test if a given edge joins two trees; and (2) to represent each tree in an $F_{i}$ in such a way that we can quickly retrieve nontree edges in $E_{i}$ which are incident to the tree. To avoid excessive cost, we explicitly maintain only those $F_{i}$ where $i$ is a multiple of $m_{i n}^{\prime 1 / 3} / \log n$. An undesirable consequence of this is that when retrieving nontree edges in $E_{i}$, other nontree edges are also retrieved.

Below, we refer to input graph vertices as "vertices" and use "node" to mean nodes of the B-tree in which we store the "ET-sequences."

ET-trees. An ET-sequence is a sequence generated from a tree by listing each vertex each time it is encountered ("an occurrence of the vertex") as a tree is searched depth-first. Each ET-sequence is stored in a B-tree of degree $d$. This allows us to implement the deletion or insertion of an edge in the forest as follows: we split a tree by deleting an edge or join two trees by inserting an edge in time $O\left(d \log _{d} n\right)$, using 
a constant number of splits and joins on the corresponding B-trees. Also we can test two vertices of the forest to determine whether they are in the same tree in time $O\left(\log _{d} n\right)$, by searching up to the roots and testing for equality. See, for example, $[1,11]$ for operations on B-trees. If $d=n^{\alpha}$, for $\alpha$ a positive constant, then the join and split operations take time $O(d)$ and the test operation takes time $O(1)$. We refer to the B-trees used to store ET-sequences as ET-trees.

This data structure allows us to keep information about a vertex so that the cumulative information about all vertices in a tree can be maintained. To do so, we make one arbitrary occurrence of each vertex a "designated" occurrence and, in each internal node of the ET-tree, we keep cumulative information about the designated occurrences in the subtree of the ET-tree rooted at that node. Here, for example, we want to know if there is at least one nontree edge incident to a vertex in a tree. We mark the vertex's designated occurrence in the ET-tree if there is a nontree edge in the graph incident to that vertex. We mark each internal node of the ET-tree if some node in its subtree is marked. We can find the endpoint of a nontree edge by starting at the root of the ET-tree and following a path of marked nodes down to a marked designated occurrence. In a degree $d$ ET-tree, each split or join operation or each change to the number associated with an occurrence requires the adjustment of $O\left(\log _{d} n\right)$ internal nodes with each adjustment taking $O(d)$ timesteps. For other applications of ET-trees, see [9].

We maintain the following data structures.

- Each edge is labelled by its level and a bit which indicates if it is a tree edge.

- Let $d_{F}=\max \left\{m_{i n}^{\prime 1 / 3} \log n, n^{\epsilon}\right\}$, for any constant $0<\epsilon \leq 1 / 3$. Each tree in $F$ is represented as an ET-sequence which is stored in a degree $d_{F}$ B-tree. Note that these B-trees have a constant number of levels, so that two vertices can be tested to determine if they lie in the same tree of $F$ in $O(1)$ time.

- Let $c=m_{i n}^{\prime 1 / 3} / \log n$. We partition consecutive levels into classes of size $c$. Each class is represented by the smallest level in the class, i.e., the level $j$ such that $c \mid j$ ("c divides $j$ "). That is, level $i$ is mapped to the class $f(i)=c\lfloor i / c\rfloor$. For each representative level $j$,

- we represent each tree in $F_{j}$ as an ET-sequence which is stored in a binary B-tree;

- for each vertex $v$, we create a list $L_{j}(v)$ which contains

(i) all nontree edges incident to $v$ which are in any level $i \in f^{-1}(j)$ and

(ii) all tree edges incident to $v$ which are in any level $i>j, i \in f^{-1}(j)$;

- we mark each designated occurrence of a vertex $v$ whose list $L_{j}(v)$ is nonempty. Each internal node of the ET-tree is marked if its subtree contains a marked occurrence.

2.2. The Search routine. Search $\left(T_{i}(u)\right)$ returns all nontree edges in level $i$ incident to $T_{i}(u)$. It begins by searching $T_{f(i)}(u)$ which is a subtree of $T_{i}(u)$. It proceeds by examining all edges in $L_{f(i)}(v)$ for all vertices $v$ in the tree being searched. Nontree edges in level $i$ are picked out and tree edges in levels $i^{\prime}, f(i)<i^{\prime} \leq i$, are followed to other trees of $F_{f(i)}$ which are then searched in turn. Note that all such tree edges lead to other trees of $F_{f(i)}$ which are subtrees of $T_{i}(u)$; and all subtrees of $T_{i}(u)$ will be found by this procedure. A phase of the algorithm consists of the examination of one edge $e$ in a list $L$.

$\operatorname{Search}\left(T_{i}(u)\right)$.

1. $S^{\prime} \leftarrow \emptyset$;

2. treelist $\leftarrow T_{f(i)}(u)$; 
3. Repeat until treelist is empty:

- Remove an ET-tree from the treelist.

- For each marked vertex $x$ in the ET-tree and for each edge $\{x, y\}$ in each $L_{f(i)}(x)$,

- if $\{x, y\}$ is a nontree edge on level $i$, add it to the set $S^{\prime}$ of edges to return;

- else if $\{x, y\}$ is a tree edge on level $l$ such that $l \leq i$, then add $T_{f(i)}(y)$ to treelist.

2.3. Analysis. Initialization. We compute the minimum spanning forest $F$, create the ET-trees for $F_{j}$, for each $j$ such that $c \mid j$, and partition the nontree edges by weight. Recall that $m^{\prime}{ }_{i n}$ is the number of nontree edges in the initial graph. Let $t$ be the number of edges in the initial minimum spanning forest. The creation of all the lists $L$ takes time proportional to the number of nontree edges $m^{\prime}{ }_{i n}$. The building of ET-trees for $F$ and all $F_{j}$ such that $c \mid j$ and the marking of internal nodes takes time proportional to the size of each forest or $O\left(\left(\left(m^{\prime}{ }_{i n} / k\right) / c\right) t+m^{\prime}{ }_{i n}\right)=O\left({m^{\prime}}_{i n}^{\prime / 3} t+m^{\prime}{ }_{i n}\right)$.

Deletions of nontree edges. Deleting a nontree edge on any level may require unmarking a designated occurrence of a vertex in some ET-tree, which may require unmarking internal nodes on the path to the root in $O(\log n)$ time.

Deletions and insertions of tree edges. Deleting a tree edge takes worst-case time $O\left(d_{F}\right)$ to delete it from the ET-tree of $F$ and worst-case time $O(\log n)$ to delete it from the ET-tree of each $F_{j}$ such that $c \mid j$, for a total of $O\left(d_{F}+\left(\left(m^{\prime}{ }_{i n} / k\right) / c\right) \log n\right)$ time per edge. Inserting a replacement edge takes the same time.

Finding a replacement edge. We first analyze the cost of Search. Let the weight $w(T)$ of a tree $T$ of some $F_{i}$ be $\sum\left|L_{f(i)}(v)\right|$ summed over all vertices $v$ in $T$. It costs $O(\log n)$ to move down the path from the root to a leaf in an ET-tree to find a marked occurrence of a vertex, or to move up a tree from an occurrence to the root. Thus, the cost of $\operatorname{Search}\left(T_{i}(x)\right)$ is $O(\log n)$ times the number of edges examined, or $O\left(w\left(T_{i}(x)\right) \log n\right)$, if Search is carried out until it ends, and $O(k)$, if it is run for $k / \log n$ phases.

In Replace $(u, v, i)$, if $w\left(T_{i}(u)\right) \leq w\left(T_{i}(v)\right)$, then we refer to $T_{i}(u)$ as the smaller component $T_{1}$; otherwise, $T_{1}$ is $T_{i}(v)$. The cost of a call to $\operatorname{Replace}(u, v, i)$ is the cost of the Search plus the cost of testing each edge in $S$. The number of edges in $S$ is $O\left(\min \left\{k, w\left(T_{1}\right)\right\}\right)$. We can use the $d_{F}$-degree ET-tree representation for $F$ to test each edge at cost $O(1)$. Thus the cost of a call to Replace is $O\left(\min \left\{k, w\left(T_{1}\right) \log n\right\}\right)$.

To pay for these costs, If a replacement edge is found on level $i$ then we charge the cost of Replace $(u, v, i)$ to the deletion. In addition, we charge the cost of modifying $F$ to the deletion so the total cost charged to the deletion is $O\left(\min \left\{k, w\left(T_{1}\right) \log n\right\}+\right.$ $\left.\left(\left(m^{\prime}{ }_{i n} / k\right) / c\right) \log n+d_{F}\right)=O\left(\left(\left(m_{i n}^{\prime} / k\right) / c\right) \log n+d_{F}\right)$.

If no replacement edge is found on level $i$ then a tree of $F_{i}$ which was split by the deletion remains split. We use the following.

Claim 2.1. $O\left(\sum w\left(T_{1}\right)\right)$ summed over all smaller components $T_{1}$ which split from a tree $T$ on any given level during all Replace operations is $O(w(T) \log n)$.

The proof of the claim follows [5]. The first time a smaller component $T_{1}$ of a tree $T$ is searched, it can have weight no greater than $w(T) / 2$. Between two successive times that $\left|L_{f(i)}(v)\right|$ contributes to the weight of a smaller component $T_{1}$ and that component splits off, the weight of a smaller component $T_{1}$ containing $v$ is no more than half its weight the previous time. Hence $\left|L_{f(i)}(v)\right|$ contributes to the weight of any $T_{1}$ no more than $\log _{2} w(T)=O(\log n)$ times. That is, $\left.O\left(\sum w\left(T_{1}\right)\right)=O\left(\sum_{v \in T}\left|L_{f(i)}(v)\right|\right) \log n\right)=O(w(T) \log n)$. 
There are at most $k$ edges per level (except for level 0 , which has at most $k$ nontree edges). Each $L_{j}(v)$ consists of edges from $c$ levels. Since level 0 tree edges do not belong to any list $L_{j}(v)$, the maximum weight of a tree $w(T)$ is $c k$. Thus the total cost charged to a level is $O\left(c k \log ^{2} n\right)$. Summing over all levels we have $O\left(\left(m^{\prime}{ }_{i n} / k\right)\left(c k \log ^{2} n\right)=O\left(m^{\prime}{ }_{i n} c \log ^{2} n\right)\right.$, or an amortized cost per deletion of $O\left(c \log ^{2} n\right)=O\left({m^{\prime}}_{i n}^{1 / 3} \log n\right)$, if $\Omega\left(m^{\prime}{ }_{i n}\right)$ edges are deleted.

The cost charged to each deletion is $O\left(\left(m^{\prime}{ }_{i n} / c k\right)(\log n)+d_{F}\right)$. For $d_{F}=\max$ $\left\{m^{\prime 1 / 3} \log n, n^{\epsilon}\right\}$ and $c=m_{i n}^{\prime 1 / 3} / \log n$, this is $O\left(m^{\prime 1 / 3} \log n+n^{\epsilon}\right)$.

To summarize the cost of initialization when amortized over $\Omega\left(m_{i n}\right)$ operations is $O\left(m_{i n}^{\prime 1 / 3}\right)$ and the cost per deletion of an edge and finding replacement edges, when amortized over $\Omega\left(m^{\prime}{ }_{i n}\right)$ operations is $O\left({m^{\prime}}_{i n}^{1 / 3} \log n+n^{\epsilon}\right)$. Thus for a sequence of $\Omega\left(m_{i n}\right)$ operations, the amortized time per update is $O\left({m^{\prime}}_{i n}^{1 / 3} \log n+n^{\epsilon}\right)$.

Finally, we note that the query of the form "Are vertices $i$ and $j$ connected?" can be answered using the ET-tree data structure for $F$ in $O(1)$ time.

3. From deletions-only to fully dynamic. In this section, we show a general technique to develop a fully dynamic data structure using several deletions-only data structures with an added operation. (We call these "extended" deletions-only data structures.) As before, we assume the edge weights are distinct.

First, we define the following operation on a deletions-only data structure $A$.

batch_add $\left(G, E^{\prime}, F^{\prime}\right)$ : Given a graph $G=(V, E)$ with minimum spanning forest $F$, insert all edges of $E^{\prime}$ into $G$, if they are not already there. The resulting spanning forest $F^{\prime}$ is given.

We refer to the period of time which occurs between two consecutive calls to batch_add on a graph $G$, or between the start of the algorithm and the first batch_add on $G$ as a period of $G$. Alternatively, a period may be terminated prematurely (see below).

We prove the following theorem.

THEOREM 3.1. Suppose for any value of $n$ and $m_{i n}^{\prime}$, there is an extended deletionsonly data structure for any dynamic graph $G=(V, E)$ with $|V|=n$ and the number of nontree edges in the edgeset $E$ is initially $m_{i n}^{\prime}$, such that $\left(n+m_{i n}^{\prime}\right) f^{0}\left(m_{i n}^{\prime}, n\right)$ is the worst case time needed to initialize $A$, and $\left(y+m_{i n}^{\prime}\right) f\left(m_{i n}^{\prime}, n\right)$ is an upper bound on the time to process y deletions.

Suppose we can process a batch_add $\left(H, E^{\prime}, F^{\prime}\right)$, following any period in which $y$ edges were deleted from $G$, in time $O\left(\left(y+m_{\text {in }}^{\prime}+\left|F^{\prime} \backslash F\right|\right) f^{B}\left(m_{i n}^{\prime}, n\right)\right)$, where $m_{i n}^{\prime}$ is an upper bound on the total number of nontree edges in $G$ after the batch_add.

We also assume that $f^{0}, f, f^{B}$ are monotone nondecreasing functions.

For any value $d$ there is a fully dynamic minimum spanning forest data structure that runs in amortized cost per edge deletion or insertion of $O\left(d \log _{d} n+\sum_{i=0}^{s}(s-\right.$ $\left.i+1)\left[f^{0}\left(2^{i}, n\right)+f\left(2^{i}, n\right)+f^{B}\left(2^{i}, n\right)\right]\right)$ where $s \leq 3+\lg m$ and $m$ is the size (vertices plus edges) of the dynamic graph at the time of the update. Here, costs are amortized over a sequence of $m_{\text {in }}$ update operations, where $m_{\text {in }}$ is the size of the initial graph.

In section 3.4, we show that the following corollary holds by choosing $d=n^{\epsilon}$ for any constant $1 / 3 \geq \epsilon>0$ and using the data structure of the previous section.

COROLlary 3.2. A minimum spanning forest can be maintained in a fully $d y$ namic graph with amortized cost per update of $O\left(m^{1 / 3} \log n\right)$, where $m$ is the size of the graph at the time of the update, for a sequence of $\Omega\left(m_{i n}\right)$ operations.

Noting that the function $f(m, n)=O\left(m^{1 / 3} \log n\right)$ is "well behaved" in the sense of Theorem 3.3.2 of [4] gives the main result of the paper. 
COROLlary 3.3. A minimum spanning forest can be maintained in a fully $d y$ namic graph with amortized cost per update of $O\left(n^{1 / 3} \log n\right)$, where $n$ is the number of vertices in the graph at the time of the update, for a sequence of $\Omega\left(m_{i n}\right)$ operations.

We prove our theorem by constructing a fully dynamic data structure from extended deletions-only data structures.

Definitions. We refer to the current minimum spanning forest of $G$ as the (global) $M S F$. Let $m^{\prime}$ be the number of nontree edges in the current graph, $m_{\text {in }}^{\prime}$ denote the number of nontree edges in the initial graph, and $m$ denote the current size (vertices and edges) of $G$.

During the course of the algorithm, we simultaneously maintain up to $s \leq$ $\max \left\{\lg n, \lg \left(4 m^{\prime}\right)\right\}$ extended deletions-only data structures $A_{0}, A_{1}, \ldots, A_{s}$, where each $A_{i}$ is an extended deletions-only minimum spanning forest data structure for a subgraph $G^{i}+\left(V, E^{i}\right)$ of the global graph $G=(V, E)$. We call this the composite data structure. We maintain each tree of the MSF as a Sleator-Tarjan dynamic tree [13] and also as an ET-tree of degree $d$.

The minimum spanning forest of $G^{i}$ as maintained by $A_{i}$ is referred to as a local spanning forest and denoted $F^{i}$. A local nontree edge of $A_{i}$ is an edge of $G^{i}$ which is not in $A_{i}$ 's local spanning forest or the MSF. We maintain $x_{i}$ to be the number of local nontree edges in $\cup_{j \leq i} A_{j}$.

When $m^{\prime}$ falls below $2^{s} / 4$ and $s>\lg n, s$ is reset and the composite data structure is reinitialized. Between two consecutive resets, we define the period of time which occurs between two consecutive calls to batch_add on a graph $G$, or between the initialization or reinitialization of the composite data structure and the first batch_add on $G$ as a period of $G$. A reset terminates all periods.

The size of a graph refers to the number of vertices plus edges.

We maintain the following invariants.

Invariant. (1) Every edge in the local forest of some $A_{i}$ is (a) in the MSF or (b) is a local nontree edge in some $A_{j}, j \neq i$.

(2) $E=\left(\cup E^{i}\right) \cup M S F$.

We now describe the algorithm.

To initialize, Let the initial value of $s=\left\lceil\lg m_{i n}^{\prime}\right\rceil$. We initialize $A_{s}$ as an extended deletions-only data structure for $G^{s}=G$ with $F^{s}=M S F$ and the set of local nontree edges being all nontree edges of $G$.

To perform an insertion operation, $\operatorname{insert}(u, v)$ is called, where $(u, v)$ is an edge to be inserted into $G$.

$\operatorname{insert}(e)$ :

1. Use the dynamic tree to determine if $e$ should be added to the MSF:

Determine if there is a path between $e$ 's endpoints in the MSF. If so, set $f$ to the maximum weight edge on the path.

2. If $e$ is lighter than $f$, remove $f$ from the MSF.

If there is no path between $e$ 's endpoints, either because $f$ has been removed or there was none previously, call insert_nontree $(f)$, and add $e$ to the MSF.

3. Else call insert_nontree $(e)$.

The following subroutine inserts a nontree edge $e$ into the composite data structure.

insert_nontree $(e)$. Let $\left.i=\min _{\{} j \mid x_{j}<2^{j}\right\}$. Let $E^{\prime}$ be the set of local nontree edges in $\cup_{j<i} E^{j} \cup\{e\}$.

1. Delete the edges of $E^{\prime}$ from $A_{j}, j<i$.

Set $x_{j}=0$. 
2. If $A_{i}$ is not initialized, initialize $A_{i}$ on the empty graph $G^{i}$ consisting of $n$ vertices and no edges.

3. Call batch_add $\left(G^{i}, E^{\prime} \cup M S F, M S F\right)$. Adjust $x_{i}$ accordingly.

After the procedure, the local nontree edges of $G^{i}$ are the nontree edges previously contained in $\cup_{j \leq i} A_{j}$. Its local forest $F^{i}=M S F$. Note that at the beginning of a period of $G^{i}, x_{j}=0$ for $j<i$.

To delete an edge $e$ from $G$ :

delete $(e)$ :

1. Delete $e$ from all data structures in which it appears, including all $G^{i}$, and update corresponding $A_{i}$ accordingly. Thus for each local spanning forest $F^{i}$ which contained $e$, the local replacement edge $e^{\prime}$ is determined, if there is one.

2. If $e$ was in the MSF, use the ET-tree representation of the tree containing $e$ in the MSF to determine which of those local replacement edges reconnect the two subtrees which result from the deletion of $e$. Insert the lightest connecting edge into the MSF.

3. All other local replacement edges are reinserted into the composite data structure using the procedure insert_nontree.

4. If $n<x_{s} \leq 2^{s-2}$, reinitialize the composite data structure. That is, set $s=\left\lceil\lg x_{s}\right\rceil$; initialize $A_{s}$ as an extended deletions-only data structure for $G^{s}=G$ with $F^{s}=M S F$ and the set of local nontree edges being all nontree edges of $G$.

3.1. Proof of correctness. It is easy to see that the invariants are maintained, by induction on the number of operations. Initially, the invariants hold since $G^{s}=G$. Invariant (2) is preserved after each insertion, since each edge when added to $G$ is either added to the MSF or some $G^{i}$. Each edge, when deleted from $G$, is deleted from all data structures in which it appears. Invariant (1) holds for $A_{i}$ when $A_{i}$ is initialized or a batch_add is executed since the local forest $F_{i}=M S F$. The local forest of $A_{i}$ changes only when an edge is deleted and is replaced by some edge $e$. Edge $e$ is then either put into the MSF or reinserted into the composite data structure. In that case, it is added to some $A_{j}$ by a batch_add operation. If $e$ is not in the MSF, then $e$ becomes a local nontree edge of $A_{j}$. In either case, invariant (1) is preserved.

The correctness of the algorithm follows easily from the invariants. We use the well-known fact that an edge is in the minimum spanning tree iff it is not the heaviest edge in any cycle ("red rule" [14]). We also note that every edge in the composite data structure is an edge in $G$.

Let $e$ be an edge of the MSF which is deleted. Let $e^{\prime}$ be the correct replacement edge. Consider the state of the composite data structures right before the deletion of $e$. By the invariant, since $e^{\prime}$ was not in the MSF, it was a local nontree edge in some $A_{i}$.

Suppose $e^{\prime}$ is a local nontree edge in $A_{i}$. Since $e^{\prime}$ is the correct replacement edge for $e$ in the MSF, then after $e^{\prime}$ 's deletion, $e^{\prime}$ is not the heaviest edge in any cycle of $G$ and therefore is not the heaviest edge of any cycle of $G^{i}$. Hence, after $e^{\prime}$ s deletion, $e^{\prime}$ becomes a local forest edge, i.e., $e^{\prime}$ is a local replacement edge for $e$ in $G^{i}$. Recall that $e^{\prime}$ is the minimum weight edge which connects the two subtrees of the MSF resulting from the deletion of $e$. Thus, $e^{\prime}$ is the lightest connecting edge from the set of local replacement edges and is chosen in Step 2 of the delete algorithm. 
3.2. Analysis. We first prove the following claims.

Claim 3.4. During any full period of $G^{i}$, there were at least $2^{i-1} /(s-i+1)$ updates to $G$.

Proof. For $i>0$, immediately before batch_add is executed on $G^{i}, x_{i-1} \geq 2^{i-1}$. Immediately afterwards, $x_{i-1}=0$.

We examine the types of insertions into the composite data structure to see how they affect $x_{i}$ : (a) when a nontree edge is inserted into $G$, (b) when an edge is replaced in the MSF after an insertion, and (c) when an edge is deleted in $G$ and it is replaced in up to $s$ local spanning forests. The first two cases cause $x_{i}$ to increase by no more than one. The third case may cause up to $s$ insertions. However, the $s$ insertions do not affect all $A_{i}$ the same. Each insertion in this case results from a local nontree edge $e$ becoming a local forest edge. Hence if this occurs in some $A_{j}, j \leq i$, the increase of $x_{i}$ resulting from the insertion of a copy of $e$ into the composite data structure is offset by the decrease of $x_{i}$ caused by the change in status of $e$ from a local nontree edge to a local tree edge. Thus $x_{s}$ is unchanged by a case-(c) insertion into the composite data structure, $x_{s-1}$ is increased by at most 1 , and in general, $x_{i}$ is increased by at most $s-i$.

Hence, at least $2^{i-1} /(s-i+1)$ insertions or deletions occurred during any full period of $G^{i}$. This concludes the proof of the claim.

We are now ready to analyze the costs of the algorithm.

Initialization. Since each $A_{i}$ is initialized once, the cost for initialization during the algorithm is $\left(n+2^{i}\right) f^{0}\left(2^{i}, n\right)$. Note that $A_{i}$ is initialized only if the number of nontree edges exceed $2^{i-1}$.

We amortize the initialization costs of the first data structure $A_{s}$ and all $A_{i}$ for $i<\lg n$ by requiring there to be $\Omega\left(m_{i n}\right)$ operations, where $m_{i n}$ is the size (vertices and edges) of the initial graph. We note that for at least half these operations, the current size of the graph $m \geq m_{i n} / 2$.

We amortize the cost of initializing $A_{i}, i \geq \lg n$ over the operations of the preceding period when at least $2^{i-1} /(s-i+1)$ operations occurred.

The cost of reinitialization of the composite data structure can be charged to the $2^{s} / 2$ deletions which must have occurred since the previous reset. Note that a reset only occurs when $2^{s}>n$, so that the initialization cost of $\left(n+2^{s-2}\right) f^{0}\left(2^{s-2}, n\right)$ results in a charge of $f^{0}\left(2^{s-2}, n\right)$ per operation.

Execution of batch_add. By assumption, $\left(y+2^{i}+\left|M S F \backslash F^{i}\right|\right) f^{B}\left(2^{i}, n\right)$ is an upper bound on the time to perform batch_add $\left(G^{i}, E^{\prime} \cup M S F \backslash F^{i}, M S F\right)$, where $y$ is the number of deletions performed on $G^{i}$ in the preceding period.

We can charge the cost of $y f^{B}\left(2^{i}, n\right)$ to the $y$ deletions for a cost of $f^{B}\left(2^{i}, n\right)$ each.

To charge the $2^{i} f^{B}\left(2^{i}, n\right)$, by the claim, batch_add is called on $G^{i}$ after at least $2^{i-1} /(s-i+1)$ insertions and deletions occurred in the preceding period. Charging the $2^{i} f^{B}\left(2^{i}, n\right)$ to those updates gives a cost per update of $(s-i+1) f^{B}\left(2^{i}, n\right)$.

To charge the $\left|M S F \backslash F^{i}\right| f^{B}\left(2^{i}, n\right)$, we note that at the start of the period, $F^{i}=M S F$, and for each $i$, each insertion or deletion in $G$ can cause at most one edge to be added to and/or one edge to be deleted from $F^{i}$. Thus we can charge the $\left|M S F \backslash F^{i}\right| f^{B}\left(2^{i}, n\right)$ to the operations in the preceding period, for a cost of $O\left(f^{B}\left(2^{i}, n\right)\right)$ each.

Performing deletions during a period of $G^{i}$. The cost of maintaining $A_{i}$ during a period containing $y$ deletions of edges in $G^{i}$ is, by assumption, bounded above by $\left(y+2^{i}\right) f\left(2^{i}, n\right)$. These costs can be charged in the same way as the costs for batch_add 
were charged to the operations of the preceding period.

In the unique case of the initial $A_{s}$, where $s=\left\lceil\lg m_{i n}^{\prime}\right\rceil$ when there was no preceding period, costs are amortized over the initial sequence of $\Omega\left(m_{i n}\right)$ deletions and insertions, as in the analysis of the initialization costs.

After a reset of $s$, the cost of performing deletions in $A_{s}$, after $A_{s}$ is reinitialized, is charged to the deletions which resulted in the reset, as in the analysis of the reinitialization costs.

Summary. For each $i$, the cost per operation is therefore $O\left((s-i+1)\left[f^{0}\left(2^{i}, n\right)+\right.\right.$ $\left.\left.f\left(2^{i}, n\right)+f^{B}\left(2^{i}, n\right)\right]\right)$.

Except for the initialization and reinitialization of $A_{s}$, we have charged operations of the preceding period for all costs incurred in the following period. Since the preceding periods occur in between resets of the value of $s$, we know that for the indices of the $A_{i}, i \leq s \leq \max \left\{\lg 4 m^{\prime}, \lg n\right\}$. Hence $s \leq 2+\lg m, m$ being the size of the graph at the time of the operation.

For the initialization and reinitialization of $A_{s}$, we charge operations which occurred when $s$ may have been smaller by 1 . Hence $s \leq 3+\lg m, m$ is the size of the graph at the time of the operation.

Each operation requires a constant number of updates in the dynamic tree data structure and the degree- $d$ ET-tree data structure storing the MSF. This takes time $O\left(d \log _{d} n\right)$.

Summing over $i$, we have of $O\left(d \log _{d} n+\sum_{i=0}^{s}(s-i+1)\left[f^{0}\left(2^{i}, n\right)+f\left(2^{i}, n\right)+\right.\right.$ $\left.f^{B}\left(2^{i}, n\right)\right]$, where $m$ is the current size of $G$ at the time of the operation, when amortized over a sequence of $m_{i n}$ update operations and $m_{i n}$ is the size of the initial graph.

3.3. Implementing batch_add. In this section, we show how a deletions-only data structure $A$ in section 2 for a graph $G$ which initially had $m_{\text {in }}^{\prime}$ nontree edges can be extended so that the operation batch_add which occurs after a sequence $\sigma$ of $y$ edge deletions can be implemented in time $O\left(\left(y+m_{\text {in }}^{\prime}+\left|F^{\prime} \backslash F\right|\right)\left(m_{\text {in }}^{\prime 1 / 3} \log n\right)\right)$.

We begin by restoring the ET-trees of $A$ to $M S F_{\text {old }}$, the minimum spanning forest of $G$ before the start of the sequence $\sigma$ of deletions. The cost of joining two ET-trees is asympotically the same as splitting them; thus the calculations of section 2 apply. For each deletion, the cost of restoration is $O\left(\left(m_{i n}^{\prime 1 / 3}\right) \log n+n^{\epsilon}\right)$.

We next transform $M S F_{\text {old }}$ to $M S F$ by again modifying the ET-trees. We remove every edge in $M S F_{\text {old }} \backslash M S F$ and insert every edge in $M S F \backslash M S F_{\text {old }}$ for a cost of $O\left({m^{\prime}}^{1 / 3} \log n+n^{\epsilon}\right)$.

To determine the transformations required, we keep a list of sorted changes which occurred since the last batch_add.

We remove all nontree edges which are stored in $A$ and sort the nontree edges of $E \cup E^{\prime}$, assign them to levels, and store them with the appropriate list $L$. The cost per edge of removing, sorting, and then storing is $O(\log n)$ per edge for the unique (binary) ET-tree in which the edge is stored.

Let $f^{\prime}(m, n)=m^{1 / 3} \log n+n^{\epsilon}$. We have shown an extended deletions-only data structure such that $O\left(\left(n+m_{i n}^{\prime}\right) f^{\prime}\left(m_{i n}, n\right)\right)$ is an upper bound on the worst case time needed to initialize A, and $O\left(\left(y+m_{i n}^{\prime}\right) f^{\prime}\left(m_{i n}, n\right)\right)$ is an upper bound on the time to process $y$ deletions.

We can process a batch_add $\left(G, E^{\prime}, F^{\prime}\right)$, following any period in which $y$ edges were deleted from $G$, in time $O\left(\left(y+m_{i n}^{\prime}+\left|F^{\prime} \backslash F\right|\right) f^{\prime}\left(m_{i n}^{\prime}, n\right)\right)$, where $m_{i n}^{\prime}$ is an upper bound on the total number of nontree edges in $G$ after the batch_add. 
3.4. Proof of corollary. Choose $d=n^{\epsilon}$ for any constant $\epsilon$ with $0<\epsilon \leq$ $1 / 3$. Substituting $f^{\prime}$ for $f^{B}, f$, and $f^{0}$, we conclude that there is a fully dynamic minimum spanning forest data structure that runs in amortized cost per edge deletion or insertion of $O\left(\sum_{i=0}^{s}(s-i+1) f^{\prime}\left(2^{i}, n\right)\right)$, where $s \leq 3+\lg m$.

Substituting for $f^{\prime}$ and $2^{i}$, we have $O\left(\sum_{i=0}^{s}(i+1)\left(2^{s-i}\right)^{1 / 3} \log n+n^{\epsilon}\right)=$ $O\left(\left(2^{s}\right)^{1 / 3} \log n+n^{\epsilon} \log n\right)=O\left(m^{1 / 3} \log n+n^{\epsilon^{\prime}}\right)$ for $\epsilon^{\prime}$ any constant.

\section{REFERENCES}

[1] T. Cormen, C. Leiserson, and R. Rivest, Introduction to Algorithms, Mit Press, Cambridge, MA, 1989, pp. 381-399.

[2] D. Eppstein, Dynamic Euclidean minimum spanning trees and extrema of binary functions, Discrete. Comput. Geom., 13 (1995), pp. 111-122.

[3] D. Eppstein, Z. Galil, and G. F. Italiano, Improved Sparsification, Tech. Report 93-20, Department of Information and Computer Science, University of California, Irvine, CA.

[4] D. Eppstein, Z. Galil, G. F. Italiano, and A. Nissenzweig, Sparsification-A technique for speeding up dynamic graph algorithms, J. ACM, 44 (1997), pp. 669-696.

[5] S. Even and Y. Shiloach, An on-line edge-deletion problem, J. ACM, 28 (1981), pp. 1-4.

[6] T. Feder And M. Mihail, Balanced matroids, in Proceedings of the 24th ACM Symposium on Theory of Computing, ACM Press, New York, 1992, pp. 26-38.

[7] G. N. Frederickson, Data structures for on-line updating of minimum spanning trees, SIAM J. Comput., 14 (1985), pp. 781-798.

[8] G. N. Frederickson and M. A. SRinivas, Algorithms and data structures for an expanded family of matroid intersection problems, SIAM J. Comput., 18 (1989), pp. 112-138.

[9] M. R. HENZINGER AND V. KInG, Randomized dynamic graph algorithms with polylogarithmic time per operation, J. ACM, 46 (1999), pp. 502-516.

[10] M. R. Henzinger AND M. Thorup, Improved sampling with applications to dynamic graph algorithms, in Proceedings of the 23rd International Colloquium on Automata, Languages, and Programming (ICALP), Lecture Notes in Comput. Sci. 1099, SpringerVerlag, New York, 1996, pp. 290-299.

[11] K. Mehlhorn, Data Structures and Algorithms 1: Sorting and Searching, Springer-Verlag, 1984.

[12] H. NAGAmochi AND T. IBARAKI, Linear time algorithms for finding a sparse $k$-connected spanning subgraph of a $k$-connected graph, Algorithmica, 7 (1992), pp. 583-596.

[13] D. D. Sleator, R. E. Tarjan, A data structure for dynamic trees, J. Comput. System Sci., 24 (1983), pp. 362-381.

[14] R. E. TARJan, Data Structures and Network Algorithms, SIAM, Philadelphia, 1983, p. 71. 